\title{
Forced convection heat transfer simulation using dissipative particle dynamics with energy conservation
}

\author{
Toru Yamada ${ }^{1, *}$, Anurag Kumar ${ }^{1}$, Asako Yutaka $^{2}$, Mohammad Faghri ${ }^{1}$ \\ * Corresponding author: Tel: +1(401)874-2247; Fax:+1(401)874-2355; Email: yamada@egr.uri.edu \\ 1: Department of Mechanical, Industrial and Systems Engineering, University of Rhode island, USA \\ 2: Department of Mechanical Engineering, Tokyo Metropolitan University, Japan
}

\begin{abstract}
Dissipative particle dynamics (DPD) with energy conservation was applied to simulate forced convection in parallel-plate channels with boundary conditions of constant wall temperature (CWT) and constant wall heat flux (CHF). DPD is a coarse-grained version of molecular dynamics. An additional governing equation for energy conservation was solved along with conventional DPD equations where inter-particle heat flux accounts for changes in mechanical and internal energies when particles interact with surrounding particles. The solution domain was considered to be two-dimensional with periodic boundary condition in the flow direction. Additional layers of particles on top and bottom of the channel were utilized to apply no-slip velocity and temperature boundary conditions. The governing equations for energy conservation were modified based on periodic fully developed velocity and temperature conditions. The results were shown via temperature profiles across the channel cross section. The Nusselt numbers were calculated from the temperature gradient at the wall using a second order accurate forward difference technique. The results agreed well with the exact solutions to within $2.3 \%$.
\end{abstract}

Keywords. Dissipative particle dynamics, Energy conservation, Forced convection, Heat transfer, Microscale, Mesoscale

\section{Introduction}

Dissipative particle dynamics (DPD) was first introduced by Hoogerbrugge and Koelman (1992). It emerged as a simple yet potentially powerful alternative for mesoscopic simulations. It is a coarse-grained version of molecular dynamics. It is a particle-based simulation method, where each particle represents a group or packet of actual molecules of the flow field. It has been applied to simulate the behavior of various complex fluids such as dynamics of colloids and suspensions (Koelman and Hoogerbrugge, 1993; Boek et al, 1997), surfactant aggregation and micelle formation (Groot, 2000; Dong et al, 2004), study of lipid bilayer and cell membrane damage (Groot and Rabone, 2001) and simple and complex fluid flow in microchannel (Fan et al, 2003; Symeonidis et al, 2006; Kumar et al, 2009; Abu-Nada et al, 2011).

The energy conservation equation for DPD was introduced by Español (1997), which enabled the modeling of heat transfer scenarios in DPD, called DPD with energy conservation (DPDe). DPDe have been used to model several heat transfer problems. Ripoll et al (1998) used dissipative particle with energy conservation for the simple case of thermal conduction. The model showed correct equilibrium fluctuations and reproduced Fourier's law. Mackie et al (1999) applied DPDe to both conduction and convection heat transfer problems in a box and the results quantitatively agreed with the conventional continuum numerical solution for incompressible system. Qiao and $\mathrm{He}$ (2007) and He and Qiao (2008) applied DPDe to heat conduction problems in nano-composite materials and nanofluids. Dimensionless DPDe parameters were expressed and the formulation was applied to heat conduction problems by Chaudhri and Lukes (2009). Abu-Nada (2010a) integrated different types of thermal boundary conditions of heat conduction problems in DPDe. The boundary conditions used in the study were combinations of constant temperature, constant heat flux, adiabatic and convective heat transfer. Abu- 
Nada (2010b) also applied DPDe to natural heat convection problems. The results were compared well with finite volume results. DPDe was mostly applied to heat conduction and there exist only a few investigations on convective heat transfer (Mackie et al, 1999; Abu-Nada, 2010b). Moreover, there is no literature available on forced convective heat transfer using DPD.

The study of forced convection heat transfer at micro and mesoscale is crucial for further development of compact devices such as micro-electromechanical systems (MEMS), integrated circuit boards, and other miniaturized devices. In the heat exchangers for these devices, coolants flow through microchannels. The enhancing techniques ( for example, additional surface by fins ) used for macroscale heat exchangers can be hardly applied in microscale because of fabrication difficulties. Therefore, other methods are required to improve the efficiency. For example, nanofluids have great potential for the enhancement of heat transfer and there exist many studies in heat transfer characteristics of nanofluids to investigate the effect of nanoparticles on heat transfer enhancement using computational fluid dynamics (CFD) (Heris et al, 2007; Raisee and Moghaddami, 2008; Bianco et al, 2009). However, CFD is not the suitable methodology to handle those two-phase fluids with micro- and nanopaticles at such a small scale. DPDe is a more suitable method and thus its application to forced convection heat transfer prompts further development of the studies on mesoscopic heat transfer, which motivates the present study.

The aim of this study was to apply DPDe to forced convection heat transfer problems and obtain generalized fully developed temperature profiles in the system. Thermal boundary conditions of constant wall temperature (CWT) and constant wall heat flux (CHF) were considered. Periodic temperature boundary conditions were applied in the flow direction via the methodology used by Patankar et al (1977). The equation of energy conservation was modified based on the methodology. The DPD method was benchmarked by simulating the heat transfer in parallelplate channels. Heating and cooling cases were implemented for both thermal boundary conditions. The fully developed temperature profiles were presented as the result of the simulation. Nusselt number was calculated from the results and compared with the exact solutions for both CWT and CHF.

\section{Methodology}

\subsection{Governing equations}

In DPD, each DPD particle represents a cluster of actual molecules of the flow field and move in Lagrangian fashion (Español and Warren, 1995; Groot and Warren, 1997). Each particle interacts with surrounding particles through a set of distance and velocity dependent forces within a certain cutoff radius. Time evolution is governed by Newton's second law and is given by,

$$
m_{i} \frac{d \vec{v}_{i}}{d t}=\vec{f}_{\text {internal }}+\vec{f}_{\text {external }},
$$

where $\vec{f}_{\text {internal }}$ is the inter-particle forces acting between DPD particles and $\vec{f}_{\text {external }}$ is the external force which is applied to each particle for the dynamics of the flow. The total momentum of the interacting particles are conserved and the system exhibits correct hydrodynamics of the flow. There are three types of forces in DPD, which are conservative, $\vec{f}_{i j}^{C}$, dissipative, $\vec{f}_{i j}^{D}$, and random forces, $\vec{f}_{i j}^{R}$, and are expressed as

$$
\begin{aligned}
\vec{f}_{i j}^{C} & =\sum_{j \neq i} a_{i j} \omega\left(r_{i j}\right) \vec{e}_{i j}, \\
\vec{f}_{i j}^{D} & =\sum_{j \neq i} \gamma_{i j} \omega^{2}\left(r_{i j}\right)\left(\vec{e}_{i j} \cdot \vec{v}_{i j}\right) \vec{e}_{i j}, \\
\vec{f}_{i j}^{R} & =\sum_{j \neq i} \sigma_{i j} \omega\left(r_{i j}\right) \zeta_{i j} \Delta t^{-1 / 2} \vec{e}_{i j} .
\end{aligned}
$$

$\vec{f}_{i j}$ represents the total force on particle $i$ due to the surrounding $j$ particles. The vector $\mathbf{r}_{i j}$ points $i$ to $j$ such that $\mathbf{r}_{i j}=\mathbf{r}_{i}-\mathbf{r}_{j}$ and $v_{i j}=$ $v_{i}-v_{j} . \vec{e}_{i j}$ is the unit vector pointing in direction from $i$ to $j$. The parameter $a_{i j}$ is the maximum repulsion between the particles. Parameters $\gamma_{i j}$ and $\sigma_{i j}$ are the strength of dissipative and random forces, respectively. 
For DPDe, an additional governing equation is required to conserve the total energy in the system. Each DPD particle interacts with surrounding particles and exchange energy through heat fluxes within the same cutoff radius as the momentum equations. The energy equation is given by Español (1997).

$$
C_{v} \frac{d T_{i}}{d t}=q_{i j},
$$

where $T_{i}$ is temperature and $C_{v}$ is the heat capacity at constant volume for DPD particle. The heat capacity of DPD particle is usually given as the dimensionless value, $\bar{C}_{v}=\frac{C_{v}}{k_{B}} \cdot q_{i j}$ is the inter-particle heat flux between DPD particles. It accounts for the heat fluxes by the changes in mechanical and internal energies. The change in mechanical energy leads to viscus heating $\left(q_{i j}^{V}\right)$. The change in internal energy consists of two terms: one corresponds to the heat transfer by a temperature difference $\left(q_{i j}^{C}\right)$ and another takes into account the fluctuations due to random heat fluxes $\left(q_{i j}^{R}\right) . q_{i j}^{V}, q_{i j}^{C}$, and $q_{i j}^{R}$ are expressed as,

$$
\begin{aligned}
q_{i j}^{V} & =\sum_{j \neq i} \frac{1}{2 C_{v}}\left[\omega ^ { 2 } ( r _ { i j } ) \left\{\gamma_{i j}\left(\vec{e}_{i j} \cdot \vec{v}_{i j}\right)^{2}\right.\right. \\
& \left.\left.-\frac{\sigma_{i j}^{2}}{m_{i}}\right\}-\sigma_{i j} \omega\left(r_{i j}\right)\left(\vec{e}_{i j} \cdot \vec{v}_{i j}\right) \zeta_{i j}\right] \\
q_{i j}^{C} & =\sum_{j \neq i} \kappa_{i j} \omega^{2}\left(r_{i j}\right)\left(\frac{1}{T_{i}}-\frac{1}{T_{j}}\right) \\
q_{i j}^{R} & =\sum_{j \neq i} \alpha_{i j} \omega\left(r_{i j}\right) \zeta_{i j}^{e} \Delta t^{-1 / 2}
\end{aligned}
$$

where $\kappa_{i j}$ and $\alpha_{i j}$ are the strength of the conductive and random heat fluxes. The weight function $\omega$ decreases monotonically with particleparticle separation distance and becomes zero beyond the cutoff radius. The same weight function was used for the momentum and energy equations and is given by,

$$
\omega\left(r_{i j}\right)=\left\{\begin{aligned}
\frac{5}{\pi}\left(1+3 \frac{r_{i j}}{r_{c}}\right) & \left(1-\frac{r_{i j}}{r_{c}}\right)^{3} \\
& :\left(r_{i j}<r_{c}\right) \\
0 & :\left(r_{i j} \geq r_{c}\right) .
\end{aligned}\right.
$$

$\zeta_{i j}$ is a random number with zero mean and unit variance. Each pair of interacting particles has a corresponding number of $\zeta_{i j}$ and $\zeta_{i j}=\zeta_{j i}$ ensures that the momentum of the interacting pair of particle is conserved. $\zeta_{i j}^{e}$ is also a random number with zero mean and unit variance. Each pair has antisymmetric number of $\zeta_{i j}^{e}$ and $\zeta_{i j}^{e}=-\zeta_{j i}^{e}$ ensures that the energy of the interacting pair of particles is conserved. $\gamma_{i j}$ and $\sigma_{i j}$ depend on the individual temperatures of each DPD particle, and is given by,

$$
\gamma_{i j}=\frac{\sigma_{i j}^{2}\left(T_{i}+T_{j}\right)}{4 k_{B} T_{i} T_{j}},
$$

where $k_{B}$ is the Boltzmann constant. Also, $\kappa_{i j}$ and $\alpha_{i j}$ are expressed as,

$$
\begin{aligned}
\kappa_{i j} & =\frac{C_{v}^{2} k_{o}\left(T_{i}+T_{j}\right)^{2}}{4 k_{B}}, \\
\alpha_{i j} & =\sqrt{2 k_{B} \kappa_{i j}},
\end{aligned}
$$

where $k_{o}$ is a constant which controls the thermal conductivity of DPD particle (Ripoll, 2002). The problem is solved in DPD units and the mass of DPD particles and the cutoff radius $r_{c}$ were set to unity in the entire study.

\subsection{Boundary conditions for forced heat con- vection problems}

In this section, the detail of the temperature boundary conditions for CWT and CHF in DPD system are presented. Patankar et al (1977) introduced a methodology of the temperature boundary conditions for the continuum momentum and energy equations, which created generalized fully developed flow and temperature profiles using periodically varying cross section. This method is integrated into DPD method as follows.

\section{Constant wall temperature}

For the case of constant wall temperature boundary condition, the fluid temperature approaches the wall temperature in the fully developed region. Therefore, the following dimensionless temperature, $\Theta_{\mathrm{cwt}}$, is introduced. 


$$
\Theta_{\mathrm{cwt}}=\frac{T-T_{w}}{T_{m}-T_{w}},
$$

where $T_{w}$ is the wall temperature and the denominator is given by,

$$
T_{m}-T_{w}=\frac{\int\left(T-T_{w}\right) u d y}{\int u d y} .
$$

For the periodic thermally developed region, the dimensionless temperature satisfies following relationship.

$$
\begin{aligned}
\Theta_{\mathrm{cwt}}(x, y) & =\Theta_{\mathrm{cwt}}(x+L, y) \\
& =\Theta_{\mathrm{cwt}}(x+2 L, y)=\ldots
\end{aligned}
$$

Therefore, the fully developed dimensionless temperature field repeats itself at subsequent positions along x-direction. The energy equation for CWT is modified based on the dimensionless temperature and expressed as

$$
\frac{d \Theta_{\mathrm{cwt}, i}}{d t}=\frac{1}{C_{v}\left(T_{m}-T_{w}\right)}\left(q_{i j}^{V}+q_{i j}^{C}+q_{i j}^{R}\right) .
$$

Note that the heat fluxes, $q_{i j}^{V}, q_{i j}^{C}$ and $q_{i j}^{R}$ are calculated based on $T$ because the temperature in Eqs. 6-8 cannot be directly replaced by $\Theta_{\text {cwt }} . T$ for each DPD particle is calculated at each time step from its dimensionless temperature using Eq. 13.

\section{Constant wall heat flux}

For the case of constant wall heat flux boundary condition, the temperature, $T$, is subdivided into two parts.

$$
T=\Omega x+\hat{T}(x, y),
$$

where the term $\Omega x$ represents the non-periodic temperature rise (or drop) which occurs in the flow direction. $\Omega$ is constant and given by

$$
\Omega=\frac{Q}{\rho_{D P D} \dot{V} C_{v} L},
$$

where $Q$ is the rate of heat addition to the fluid in the period of $L . \rho_{D P D}$ is the number density of DPD particles in a unit cell and $\dot{V}$ is volume flow rate. $\hat{T}$ is a periodic function from module to module which satisfies,

$$
\hat{T}(x, y)=\hat{T}(x+L, y)=\hat{T}(x+2 L, y)=\ldots
$$

Equation 5 can be rewritten using $\hat{T}$ and is given by,

$$
\frac{d \hat{T}_{i}}{d t}=\frac{1}{C_{v}}\left(q_{i j}^{V}+q_{i j}^{C}+q_{i j}^{R}\right)-v_{i} \Omega,
$$

where $v_{i} \Omega$ is the source-term arising from the assumption of constant wall heat flux boundary condition. The heat fluxes, $q_{i j}^{V}, q_{i j}^{C}$ and $q_{i j}^{R}$ are calculated based on $T$, which is from the periodic temperature, $\hat{T}$, using Eq. 17.

\section{Results and discussion}

\subsection{Validation of the methodology}

The DPDe code was first validated by simulating a scenario of unsteady heat conduction in a one-dimensional slab with temperatures of $T_{C}$ and $T_{H}$. The schematic of the solution domain is shown in Fig. 1 (a). DPD particles are randomly distributed in a 2D lattice of $4 \times 30(x \times y)$. The particles were enclosed with infinite walls with constant temperatures. The walls were created by freezing the particles with a constant temperature in the extra layer of the domain. Periodic boundary condition was imposed in $\mathrm{x}-$ direction. The temperatures at top $\left(T_{H}\right)$ and bottom $\left(T_{C}\right)$ walls were maintained at 2.0 and 1.0, respectively. The time step was taken to be 0.0005 . By this means, the temperature profiles at different times were obtained by averaging the values. The simulation with lower time steps was also conducted with no effect on the results.

The initial temperature of the DPD particles was selected to be the average of the temperatures of the bottom and top walls $\left(T_{\text {initial }}=\right.$ 1.5). The dimensionless heat capacity $\left(\bar{C}_{v}\right)$ was fixed to be $1.0 \times 10^{5}$ (Qiao and $\mathrm{He}, 2007$ ) and the number density was 4 . $k_{o}$ was selected to be $1.26 \times 10^{-4}$. The analytical solution of this problem is given by Carslaw and Jaeger (1959), 


$$
\begin{aligned}
T(y, t) & =T_{C}+\left(T_{H}-T_{C}\right) \frac{y}{l} \\
& +\frac{2}{\pi} \sum_{n=1}^{\infty}\left[\frac{T_{H} \cos (n \pi)-T_{C}}{n}\right. \\
& \left.\times \sin \frac{n \pi y}{l} \exp \left(-\frac{D n^{2} \pi^{2} t}{l^{2}}\right)\right] \\
& -\frac{2 T_{\text {initial }}}{l \pi} \sum_{n=1}^{\infty}\left[\frac{\cos (n \pi)-1}{n}\right. \\
& \left.\times \sin \frac{n \pi y}{l} \exp \left(-\frac{D n^{2} \pi^{2} t}{l^{2}}\right)\right]
\end{aligned}
$$

where $l$ is the distance between top and bottom walls and $D$ is thermal diffusivity. The temperature profiles along y-direction of the system with different times $(t=0.5,5.0,15,100)$ are given in Fig. 1 (b), where the dot symbols and lines show DPD and analytical results, respectively. As shown in the figure, the temperature profiles is developed to steady state (linear profile) as time advances for both DPD and analytical results. The result of the temperature evolution for DPDe simulation is in a good agreement with the analytical solution.

\subsection{Forced convection heat transfer problems}

After the validation of the methodology, forced convection heat transfer problems were implemented. The problem considered was two dimensional parallel-plate channels with height, $M$, and length , $L$, respectively. The solution domain was divided into 4 and 40 cells in the $x$ and $y$ directions, respectively. The schematics of the domain and boundary conditions for flow, CWT and CHF are shown in Fig. 2. The parameters used in the problems are given in Table 1 . Note that $a_{i j}$ and $\sigma_{i j}$ are constant, while the other DPD parameters $\left(\gamma_{i j}, \kappa_{i j}, \alpha_{i j}\right)$ were calculated based on Eqs. $10-12$ as the temperature of each DPD particle changes at each time step. The initial temperature of fluid particle was fixed at 1.0 for both CWT and CHF.

In order to obtain a Poiseuille flow in the channel, an external force, $f_{\text {external }}=0.01$, was applied to each particle in the axial direction.

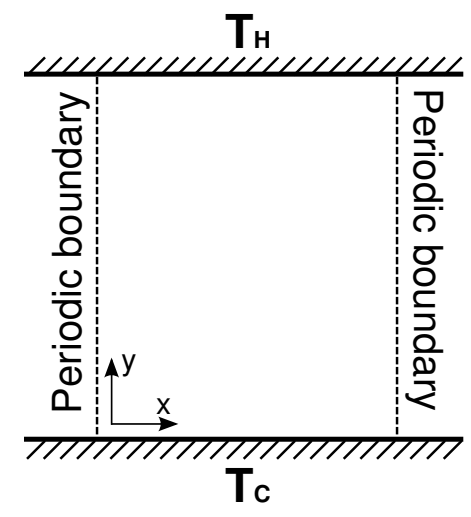

(a)

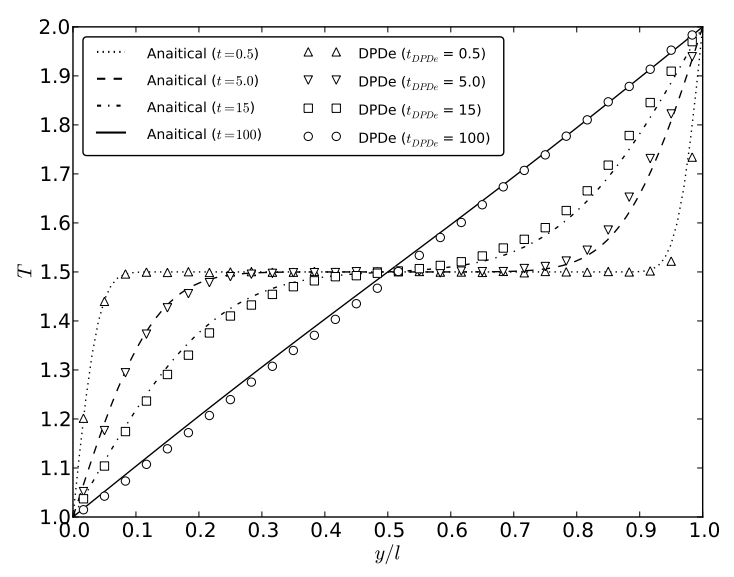

(b)

Figure 1: (a) The schematic of the solution domain for 1D slab conduction and (b) Temperature evolution: Comparison between DPDe method and analytical solution

No-slip was applied at the top and bottom of the domain and a periodic boundary condition was imposed at the left and right faces $(\mathrm{Ku}-$ mar et al, 2009). The computation was first performed with constant fluid temperature until the fully developed flow field was established (about 250,000 time steps).

\section{Constant wall temperature}

A constant wall temperature was applied to top and bottom walls and periodic boundary conditions were imposed at left and right boundaries using the methodology. In order to verify that the method can be used for both heating and cooling cases, the simulations were performed for two different wall temperatures, 1.2 (heating) and 0.8 (cooling). For these cases, 


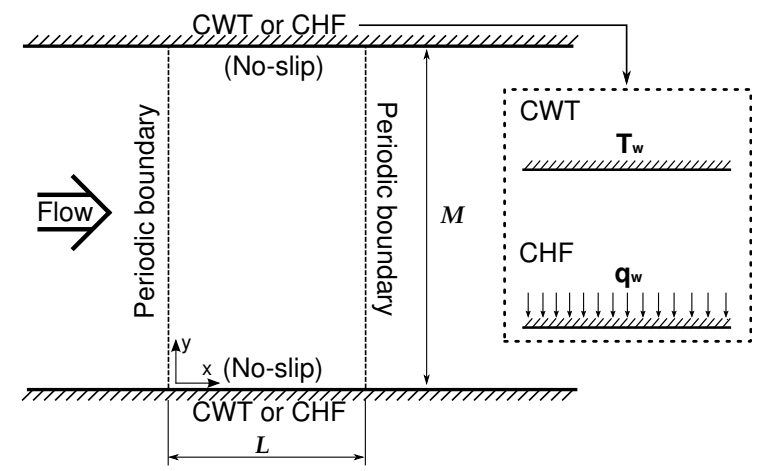

Figure 2: The schematics of the solution domain and boundary conditions for flow and temperature (CWT or CHF)

Table 1: DPDe parameters for CWT and CHF

\begin{tabular}{lc}
\hline \hline$a_{i j}$ & 18.75 \\
$\sigma_{i j}$ & 3.0 \\
$\rho_{D P D}$ & 4 \\
$\bar{C}_{v}$ & $1.0 \times 10^{5}$ \\
$k_{o}$ & $1.26 \times 10^{-4}$ \\
Total number of iterations & $1.5 \times 10^{6}$ \\
Number of iteration for averaging & $1.0 \times 10^{5}$ \\
$T_{w}(\mathrm{CWT})$ & $0.8,1.2$ \\
$q_{w}(\mathrm{CHF})$ & $-1000,1000$ \\
Time step $(\Delta t)$ & 0.01 \\
\hline \hline
\end{tabular}

the dimensionless temperature profile across the channel cross section is the same regardless of the wall temperature.

The dimensionless temperature distribution is shown in Fig. 3. As can be seen in this figure, the fully developed temperature regime for heating and cooling cases coincides with each other.

Nusselt number $(\mathrm{Nu})$ is an important parameter for forced convection heat transfer, which is described as the ratio of convective to conductive heat transfer across the boundary. Nusselt numbers for heating and cooling cases were calculated from the profile using following equation.

$$
\mathrm{Nu}_{\mathrm{cwt}}=\frac{h D_{h}}{k}=D_{h}\left(-\frac{d \Theta_{\mathrm{cwt}}}{d y}\right)_{\text {wall }} .
$$

The calculated $\mathrm{Nu}$ for heating and cooling

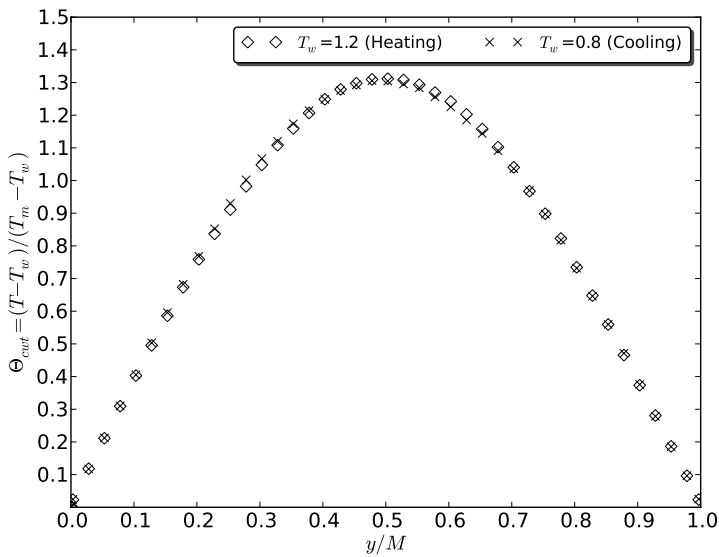

Figure 3: Dimensionless temperature distribution across the channel cross section (CWT)

cases were 7.42 and 7.71 , respectively, which are in a good agreement with the analytical value of 7.54 within $2.3 \%$.

\section{Constant wall heat flux}

For constant wall heat flux boundary condition, a constant wall heat flux was applied to top and bottom walls and periodic boundary condition was imposed at left and right boundaries. The wall temperature was not constant and was calculated at each time step using a second order forward difference formula (Abu-Nada, 2010a). It was obtained based on the first and second adjacent cells to the wall boundary as was given by the following equation.

$$
T_{w}=\frac{2 \Delta y\left(\frac{q_{w}}{k}\right)+4 T_{1}-T_{2}}{3} .
$$

where $\Delta y$ is the width of unit cell and the subscripts , 1 and 2, describe the first and second adjacent cells to the wall, respectively. Similar to the CWT problems, two different cases, heating and cooling, were simulated. The wall heat fluxes for those cases were fixed at 1000 and 1000 , respectively.

In order to compare the temperature profiles for heating and cooling cases, the calculated temperature was normalized by the following equation.

$$
\Theta_{\mathrm{chf}}=\frac{\hat{T}_{w}-\hat{T}}{q_{w} D_{h} / k}
$$




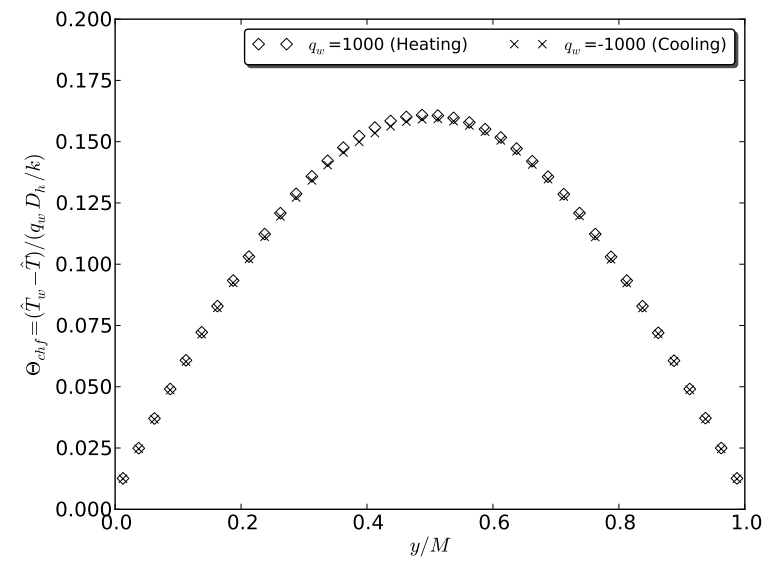

Figure 4: Dimensionless temperature distribution across the channel cross section (CHF)

where $q_{w}$ and $D_{h}$ are constant wall heat flux and hydraulic diameter (i.e $D_{h}=2 M$ for a parallelplate channel). $k$ is thermal conductivity and was calculated to be $3.6 \times 10^{5}$ (Ripoll, 2002). Figure 4 shows the normalized temperature across the channel. As can be seen in this figure, the profiles for heating and cooling cases agreed well with each other. Nusselt number was also calculated from the profile using following equation.

$$
\mathrm{Nu}_{\mathrm{chf}}=\frac{h D_{h}}{k}=\frac{D_{h}}{\hat{T}_{w}-\hat{T}_{m}}\left(-\frac{d \hat{T}}{d y}\right)_{\text {wall }} .
$$

The calculated Nusselt numbers for heating and cooling cases were 8.05 and 8.06, respectively, which are in good agreement with the analytical value of 8.235 within $2.2 \%$.

\section{Conclusions}

Forced convection heat transfer with temperature boundary conditions of constant wall temperature (CWT) and constant wall heat flux (CHF) in parallel-plate channels was simulated using dissipative particle dynamics (DPD). Periodic temperature boundary conditions were applied in the flow direction via the methodology used in the literature. The governing equation for energy conservation was modified based on the method. The DPD methodology was benchmarked by comparing Nusselt number of the numerical results with the analytical solution. The DPD results agreed well with the analytical solutions for both CWT and CHF to within $2.3 \%$.

\section{Acknowledgment}

This work is supported by the National Science Foundation grant (NSF-OISE-0530203).

\section{References}

Abu-Nada E (2010a) Modeling of Various Heat Transfer Problems Using Dissipative Particle Dynamics. Numerical Heat Transfer, Part A: Applications 58(8):660-679

Abu-Nada E (2010b) Natural convection heat transfer simulation using energy conservative dissipative particle dynamics. Physical review E, Statistical, nonlinear, and soft matter physics $81(5)$

Abu-Nada E, Kumar A, Asako Y, Faghri M (2011) Dissipative particle dynamics for complex geometries using non-orthogonal transformation. International Journal for $\mathrm{Nu}-$ merical Methods in Fluids

Bianco V, Chiacchio F, Manca O, Nardini S (2009) Numerical investigation of nanofluids forced convection in circular tubes. Applied Thermal Engineering 29(17-18):3632-3642

Boek E, Coveney P, Lekkerkerker H, Van Der Schoot P (1997) Simulating the rheology of dense colloidal suspensions using dissipative particle dynamics. Physical Review E 55(3):3124-3133

Carslaw H, Jaeger J (1959) Conduction of heat in solids. Oxford University Press

Chaudhri A, Lukes J (2009) Multicomponent Energy Conserving Dissipative Particle Dynamics: A General Framework for Mesoscopic Heat Transfer Applications. Journal of Heat Transfer 131:033,108 
Dong F, Li Y, Zhang P (2004) Mesoscopic simulation study on the orientation of surfactants adsorbed at the liquid/liquid interface. Chemical Physics Letters 399(1-3):215-219

Español P (1997) Dissipative particle dynamics with energy conservation. EPL (Europhysics Letters) 40:631

Español P, Warren P (1995) Statistical mechanics of dissipative particle dynamics. EPL (Europhysics Letters) 30:191-196

Fan X, Phan-Thien N, Yong N, Wu X, Xu D (2003) Microchannel flow of a macromolecular suspension. Physics of fluids 15:11

Groot R (2000) Mesoscopic Simulation of Polymer- Surfactant Aggregation. Langmuir 16(19):7493-7502

Groot R, Rabone K (2001) Mesoscopic simulation of cell membrane damage, morphology change and rupture by nonionic surfactants. Biophysical Journal 81(2):725-736

Groot R, Warren P (1997) Dissipative particle dynamics: Bridging the gap between atomistic and mesoscopic simulation. Journal of Chemical Physics 107(11):4423

He P, Qiao R (2008) Self-consistent fluctuating hydrodynamics simulations of thermal transport in nanoparticle suspensions. Journal of Applied Physics 103:094,305

Heris S, Esfahany M, Etemad G (2007) Numerical investigation of nanofluid laminar convective heat transfer through a circular tube. Numerical Heat Transfer Part A: Applications 52(11):1043-1058

Hoogerbrugge P, Koelman J (1992) Simulating microscopic hydrodynamic phenomena with dissipative particle dynamics. EPL (Europhysics Letters) 19:155

Koelman J, Hoogerbrugge P (1993) Dynamic simulations of hard-sphere suspensions under steady shear. EPL (Europhysics Letters) $21: 363$
Kumar A, Asako Y, Abu-Nada E, Krafczyk M, Faghri M (2009) From dissipative particle dynamics scales to physical scales: a coarse-graining study for water flow in microchannel. Microfluidics and Nanofluidics 7(4):467-477

Mackie A, Avalos J, Navas V (1999) Dissipative particle dynamics with energy conservation: Modelling of heat flow. Physical Chemistry Chemical Physics 1(9):2039-2049

Patankar S, Liu C, Sparrow E (1977) Fully developed flow and heat transfer in ducts having streamwise-periodic variations of crosssectional area. Journal of Heat Transfer 99:180

Qiao R, He P (2007) Simulation of heat conduction in nanocomposite using energyconserving dissipative particle dynamics. Molecular Simulation 33(8):677-683

Raisee M, Moghaddami M (2008) Numerical investigation of laminar forced convection of nanofluids through circular pipes. Journal of Enhanced Heat Transfer 15(4)

Ripoll M (2002) Kinetic theory of dissipative particle dynamics models. $\mathrm{PhD}$ thesis, Universidad Nacional de Educacion a Distancia, Madrid

Ripoll M, Español P, Ernst M (1998) Dissipative particle dynamics with energy conservation: Heat conduction. International Journal of Modern Physics C (IJMPC) 9:1329

Symeonidis V, Karniadakis G, Caswell B (2006) Schmidt number effects in dissipative particle dynamics simulation of polymers. The Journal of chemical physics 125:184,902 